\title{
Reconstruction of droughts in India using multiple land surface models (1951-2015)
}

Vimal Mishra ${ }^{1}$, Reepal Shah ${ }^{1}$, Syed Azhar ${ }^{1}$, Harsh Shah ${ }^{1}$, Parth Modi ${ }^{1}$, Rohini Kumar ${ }^{2}$

${ }^{1}$ Civil Engineering, Indian Institute of Technology (IIT) Gandhinagar, Gujarat, 382355

${ }^{2}$ UFZ-Helmholtz Centre for Environmental Research, Leipzig, Germany

Correspondence to: Vimal Mishra (vmishra@iitgn.ac.in) 


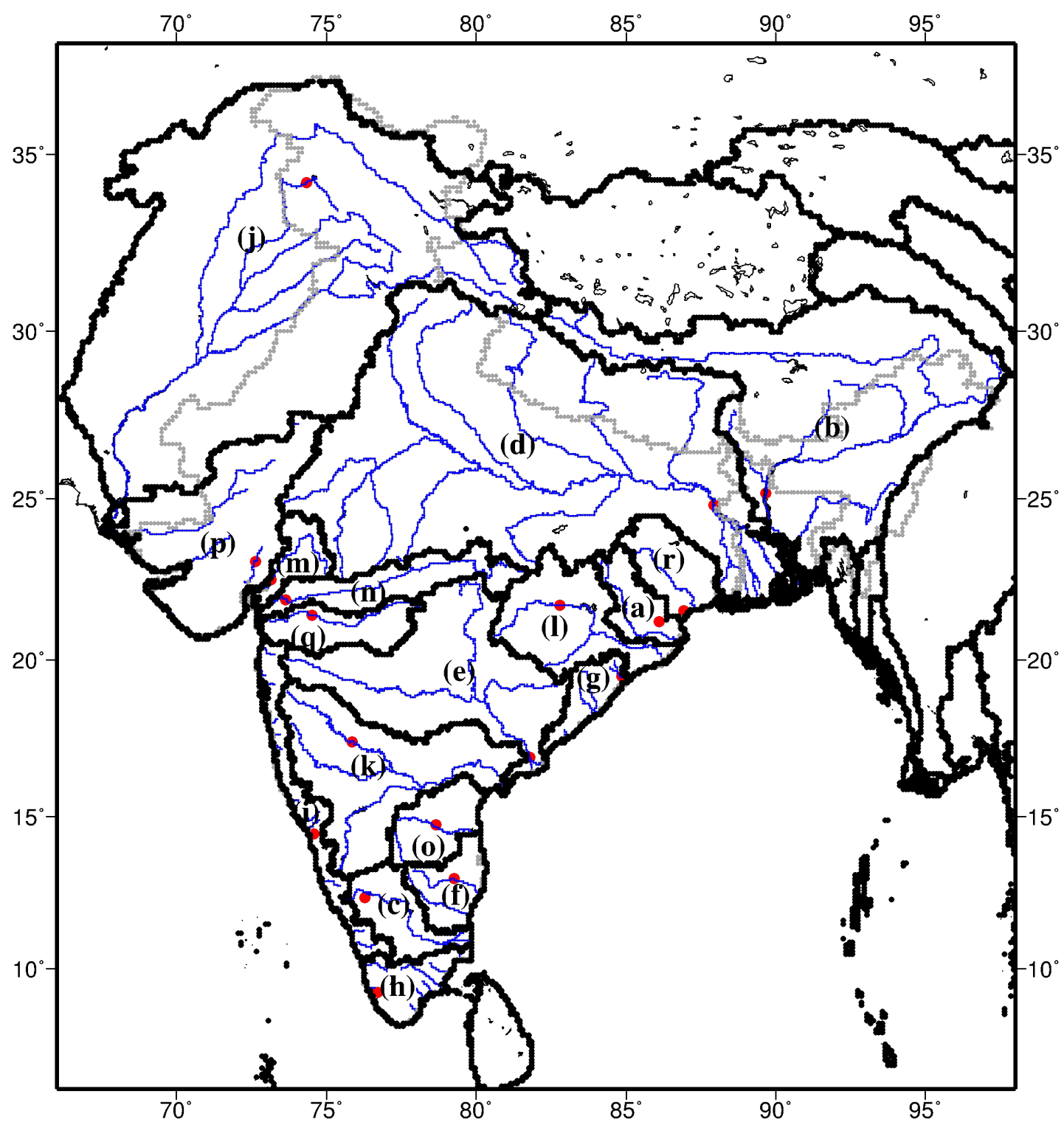

Figure S1: Map of gauge stations used for calibration and validations of models (shown with red color filled circle), river network (shown with blue color line) and basins (bordered with black color line, Refer basins name in Table 2) of India. Boundary of India is shown with grey color. 

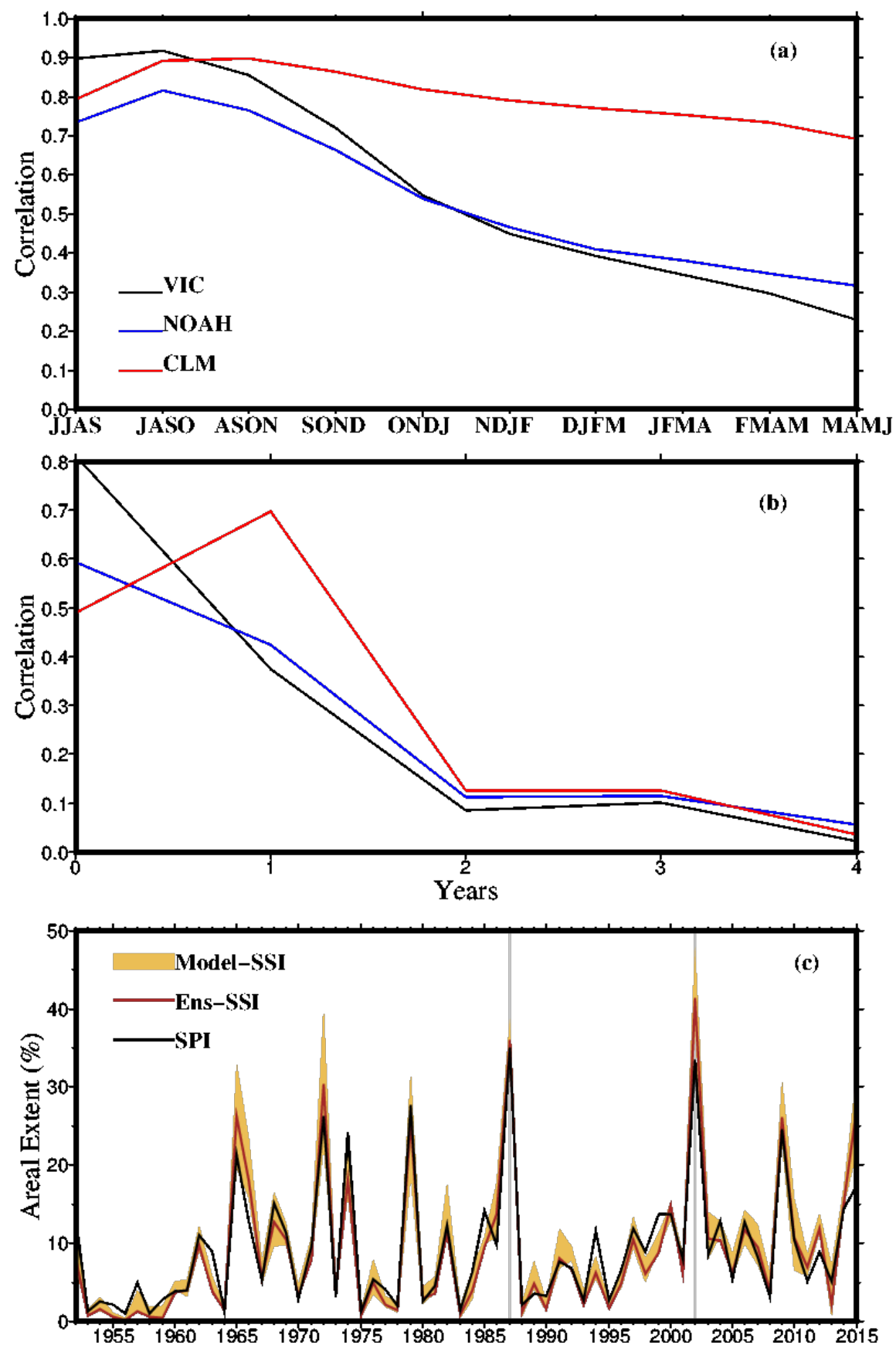

Figure S2: (a) Correlation between 4-month SPI at the end of the monsoon season (JJAS) and 4month SSI (root-zone soil moisture) at different leads. (b) Correlation of 12-month SPI at the end of December with 12-month SSI at lead varying from 0-48 months. (c) uncertainty in areal extent of root-zone soil moisture drought based on the lead time for which 4-month SSI shows the highest correlation with the 4-month SPI at the end of the monsoon season. Black line in (c) shows 4-month SPI at the end of the monsoon season. Shaded area shows indermodel uncertainty estimated using one standard deviation. Gray lines show major drought events. 




Figure S3: Reconstruction of the Rabi season (NDJF) drought events of (a-e) 1966, (f-j) 1973, (k-o) 2001, and (p-t) 2003 estimated based on (b,g,l,q) 4-month SSI at the end of February estimated using root-zone soil moisture from the VIC model, (c,h,m,r) 4-month SSI simulated using the Noah model, $(\mathrm{d}, \mathrm{i}, \mathrm{n}, \mathrm{s})$ 4-month SSI simulated using the CLM and (e,j,o,t) ensemble mean of 4-month SSI simulated using the VIC, Noah and CLM. (a,f,k,p) Air temperature anomaly during the Rabi season for the respective years. 

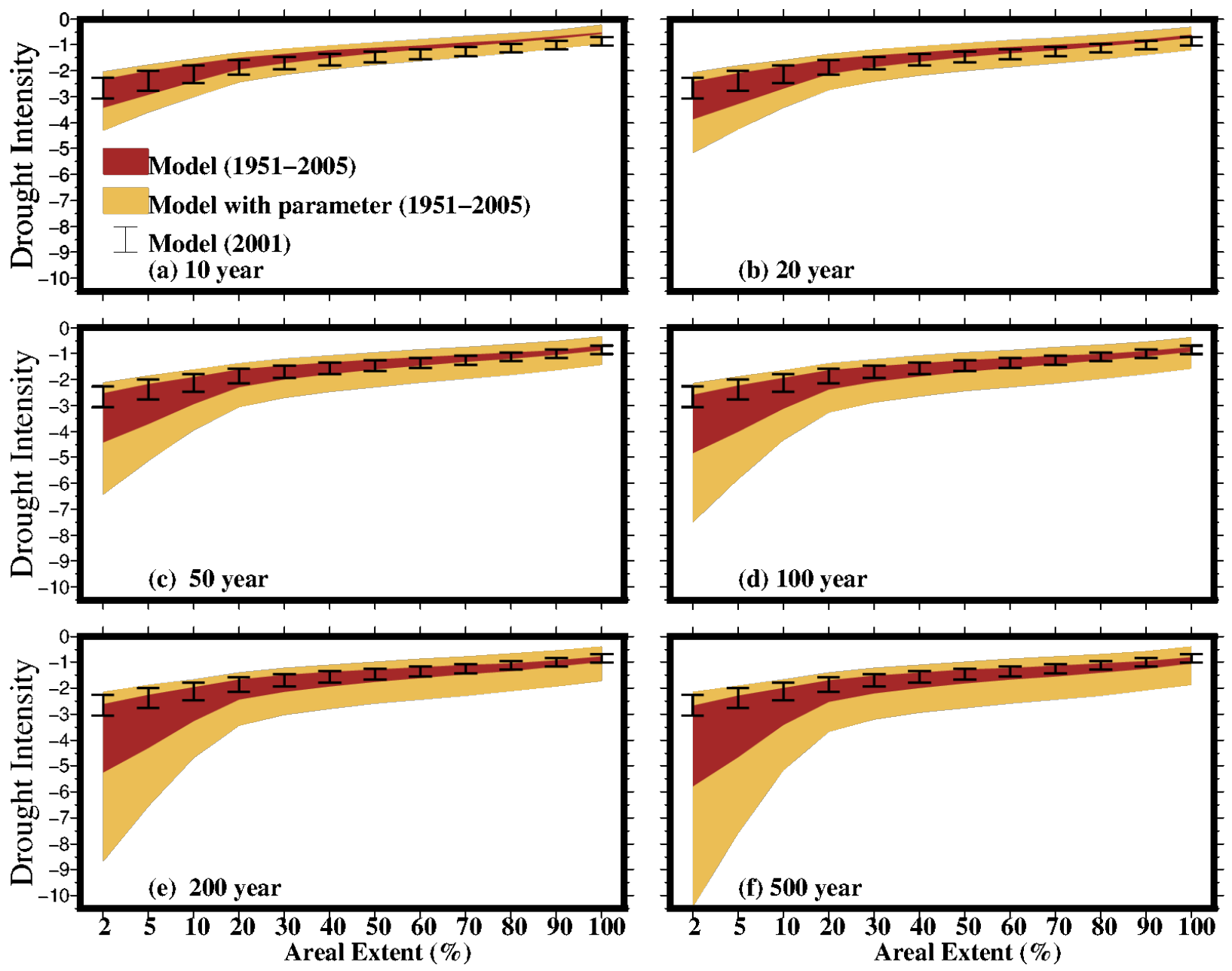

Figure S4: Uncertainty in Intensity-Areal Extent [\%]-Frequency [IAF] curve of drought during Rabi season (November through January) estimated using three LSMs [Dark brown color shade shows uncertainty in models without considering distribution uncertainty while light brown color with considering distribution uncertainty] for all-India with return periods (a) 10, (b) 20, (c) 50, (d) 100, (e) 200, and (f) 500 years. Black error-bars indicate uncertainty Intensity-Areal Extent [\%] for 2001 Rabi season drought using three LSMs. 

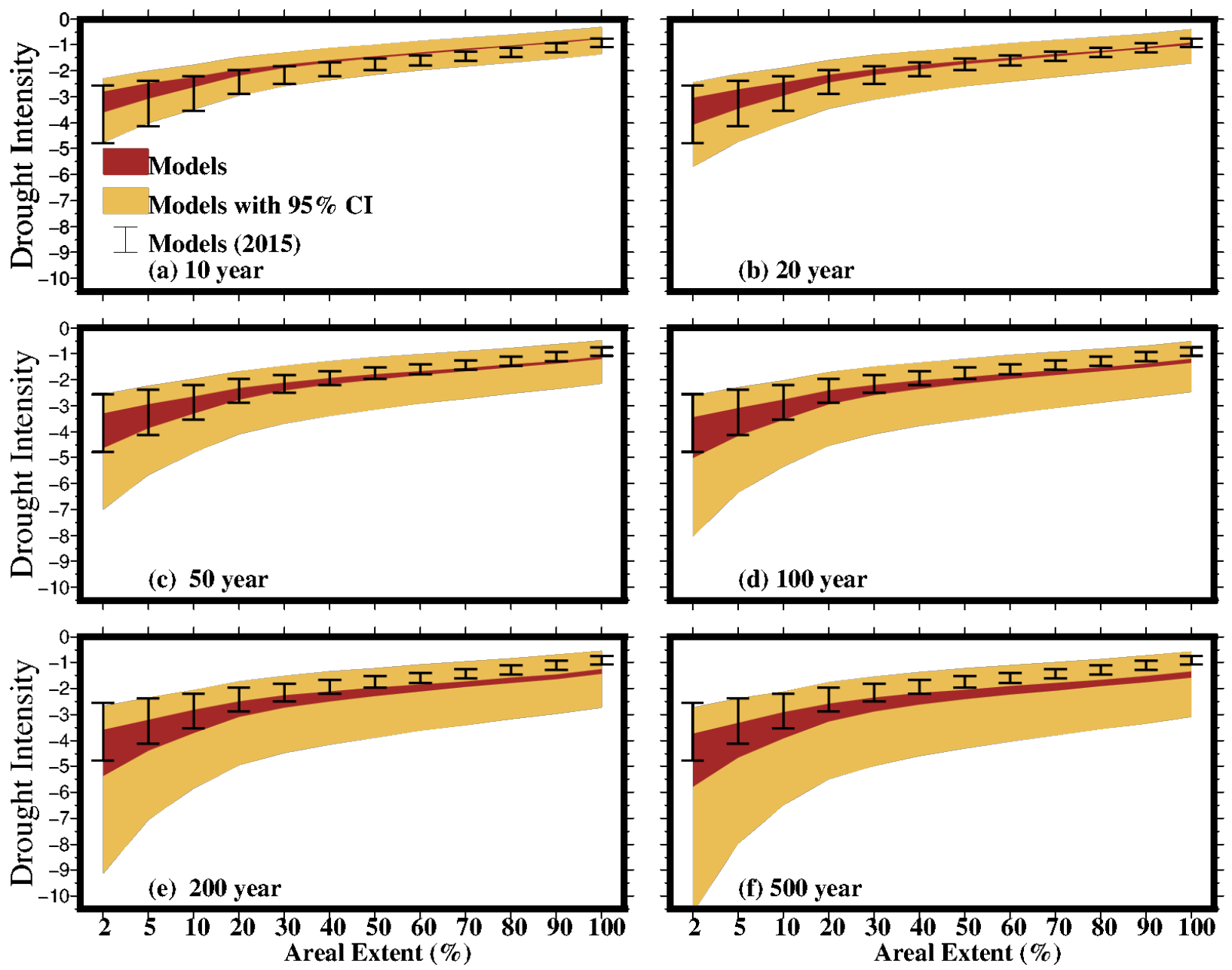

Figure S5: Same as Fig. 4 but for 12-month SSI at the end of December for the Indo-Gangetic Plain. 

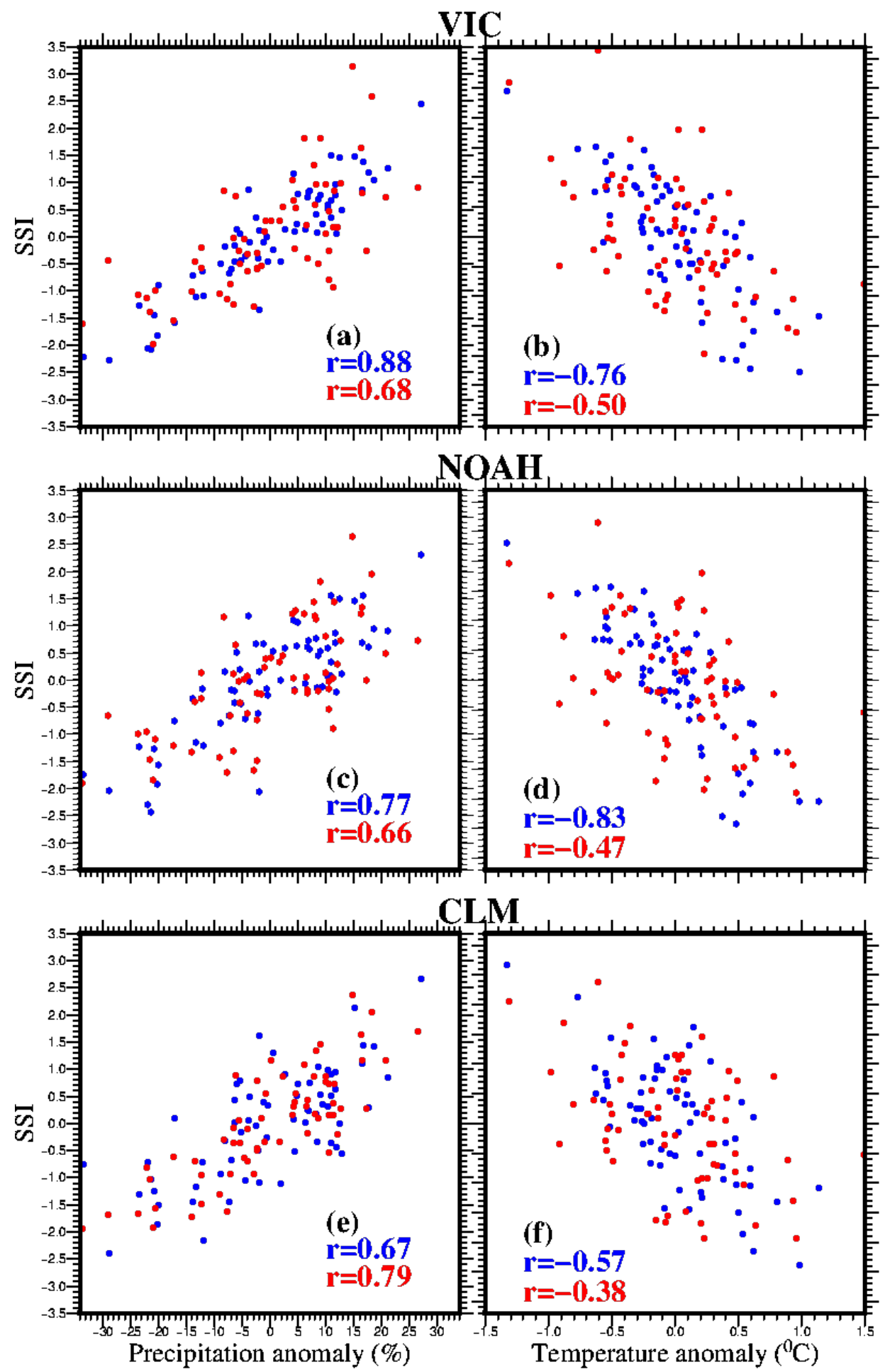

Figure S6. (a,c,e)Relationship between monsoon season precipitation anomaly (\%) and 4-month SSI at the end of the Rabi season and $(b, d, f)$ same as $(a, c, e)$ but for the relationship between 4month SSI and air temperature anomaly of the Rabi season. Correlation coefficients are shown for all-India SSI (blue) and 4-month SSI of the Indo-Gengatic Plain (red). 


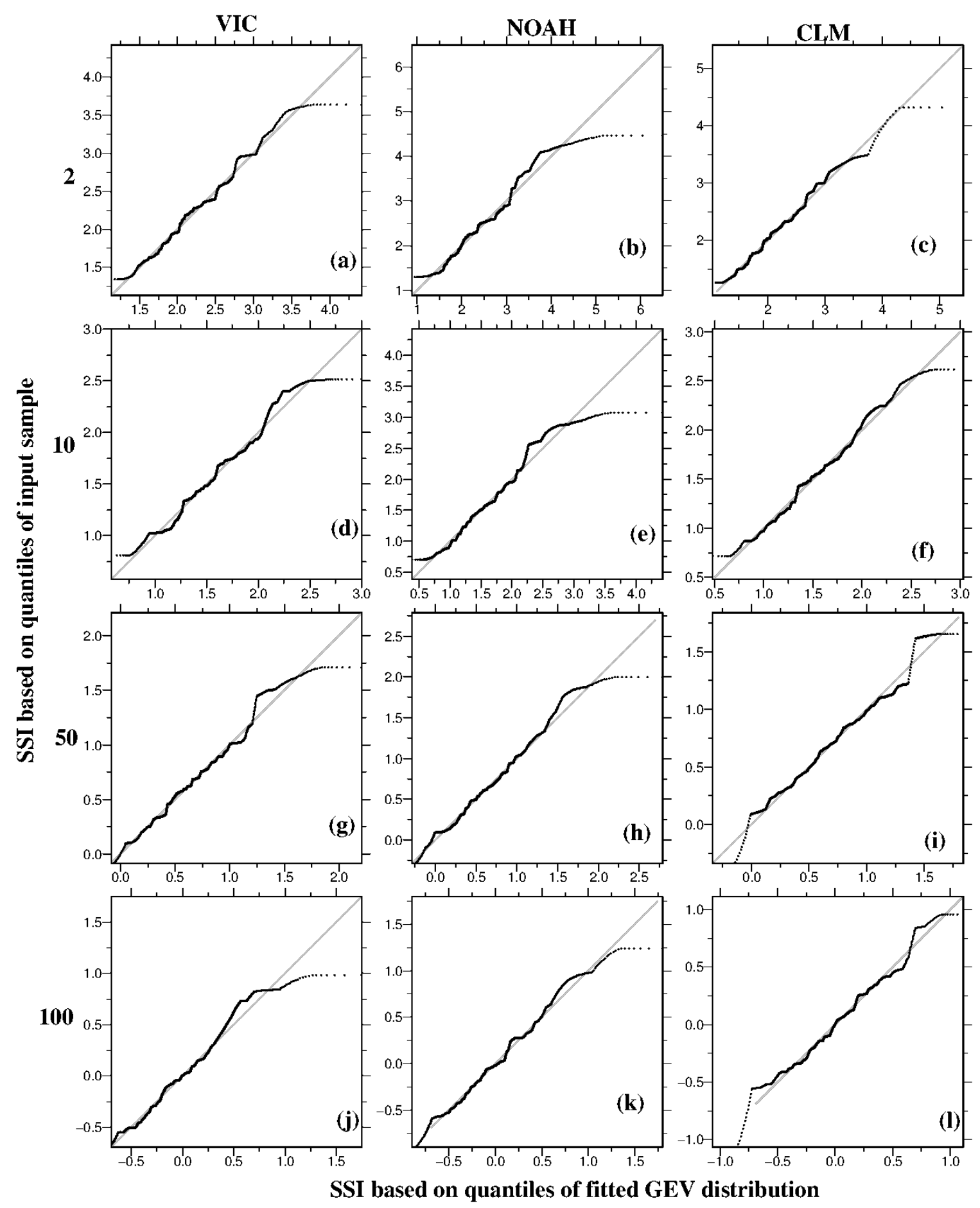

Figure S7: Quantile-quantile plot of the monsoon season SSI for different areal extent (\%, written in left) of whole India from the VIC (a,d,g,j), NOAH (b,e,h,k), and CLM (c,f,i,l) and corresponding SSI estimated by fitting GEV distribution to the monsoon season SSI. 


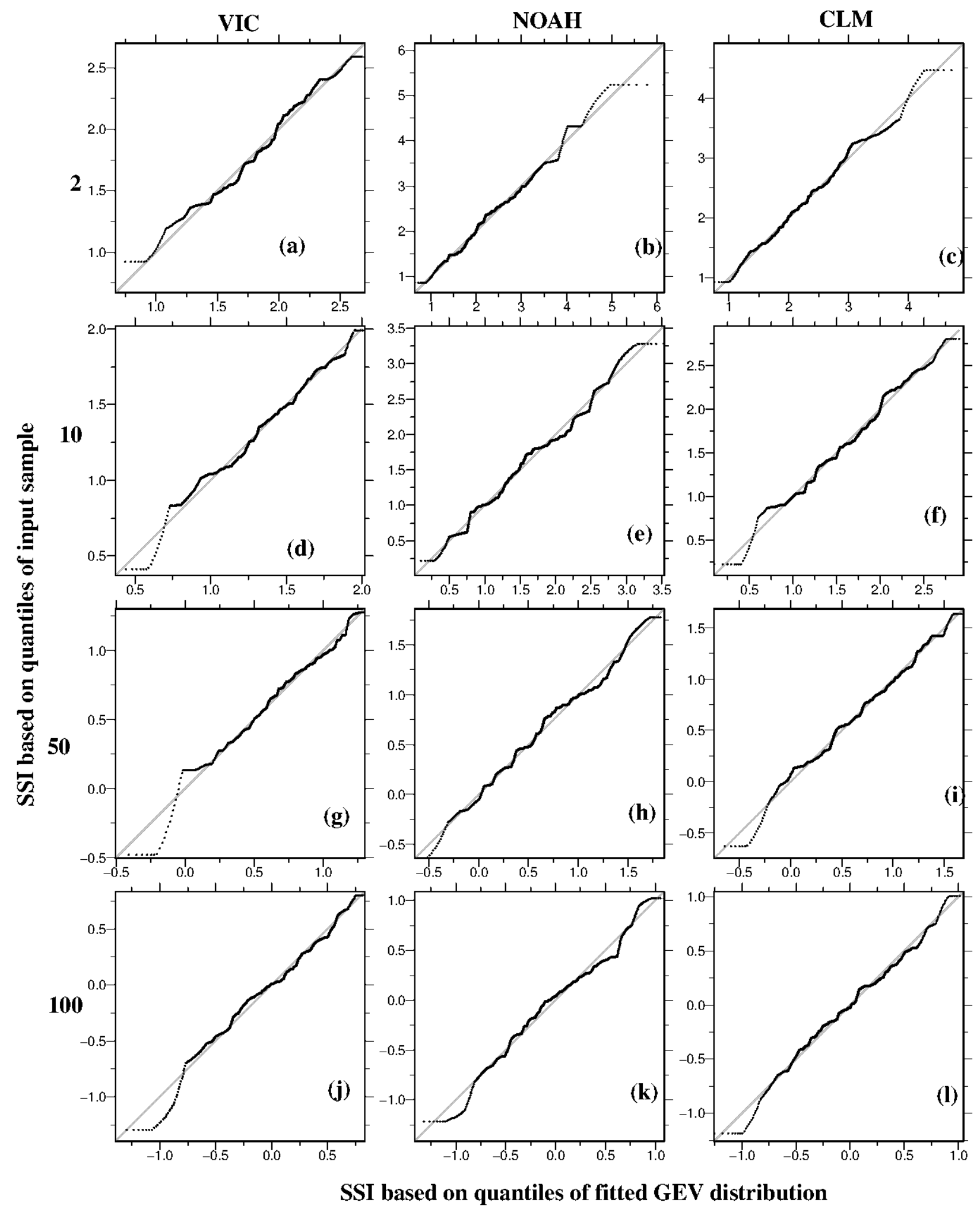

Figure S8: same as Figure S7 but for the Rabi season. 


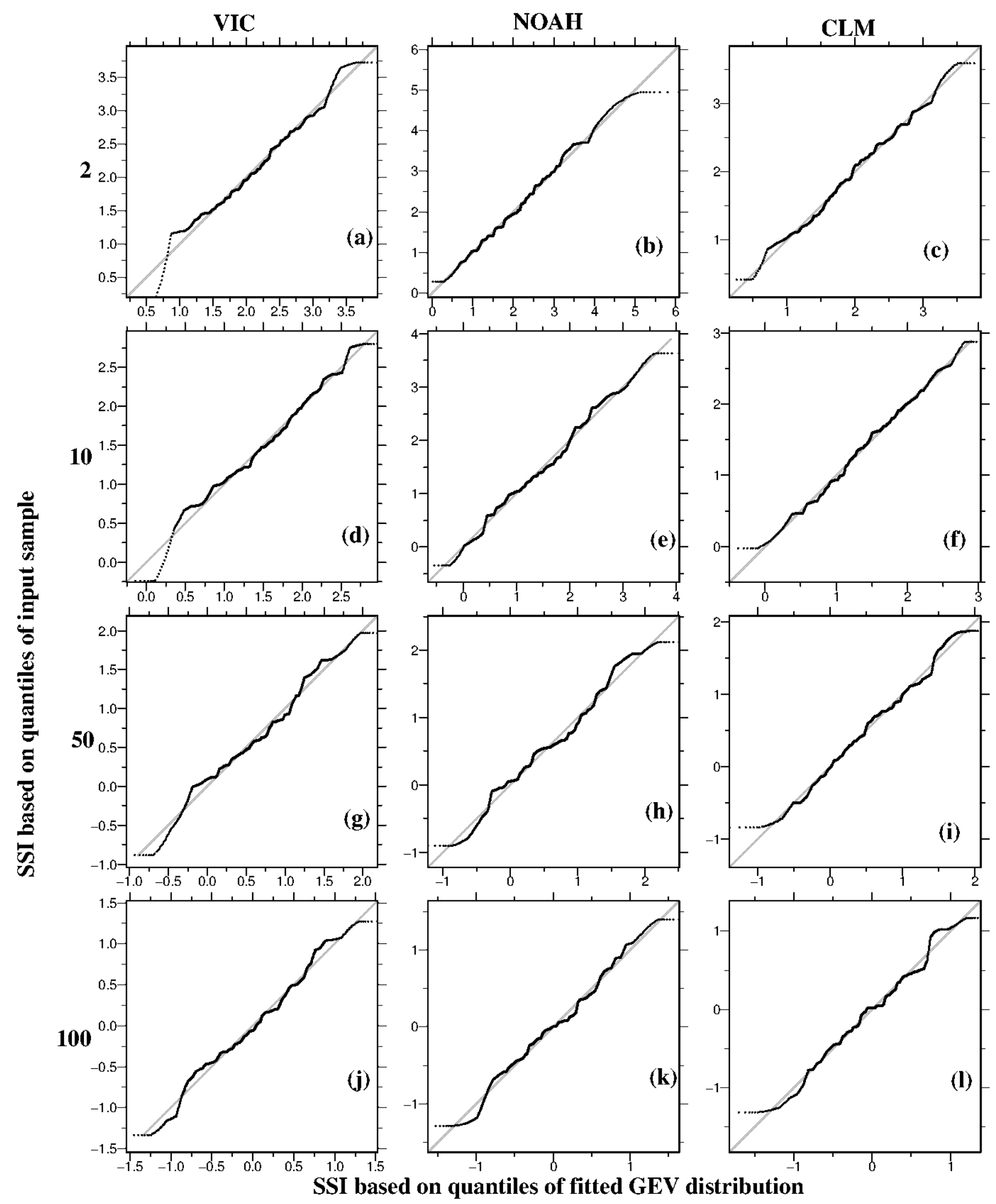

Figure S9: same as Figure S8 but for annual SSI estimated for areal extent of Indo-Gangetic plain. 
Table S1: Description of hydrological process and parameters use in all three models.

\begin{tabular}{|c|c|c|c|c|}
\hline LSM & Hydrology & \begin{tabular}{|l|} 
Soil \\
Layers
\end{tabular} & Soil/ vegetation parameters & Input Parameters \\
\hline $\begin{array}{l}\text { VIC } \\
\text { v4.2.1.a }\end{array}$ & $\begin{array}{l}\text { Variable Infiltration Capacity } \\
\text { curve for runoff, Arno model for } \\
\text { Base flow, drainage driven by } \\
\text { gravity, Penman-Monteith } \\
\text { equation for evapotranspiration } \\
\text { (ET) }\end{array}$ & $\begin{array}{l}\text { Three } \\
\text { layers }\end{array}$ & $\begin{array}{l}\text { Vegetation parameter based on } \\
1 \mathrm{~km} \text { Advanced Very High } \\
\text { Resolution Radiometer } \\
\text { (AVHRR), } \\
\text { Soil parameter from Harmonized } \\
\text { world soil database (HWSD) }\end{array}$ & $\begin{array}{l}\text { Four parameters, Precipitation, } \\
\text { Maximum and Minimum air } \\
\text { temperatures, and Wind speed }\end{array}$ \\
\hline $\begin{array}{l}\text { NOAH } \\
\text { v3.1 }\end{array}$ & $\begin{array}{l}\text { Exponential distribution of } \\
\text { infiltration capacity for runoff, } \\
\text { baseflow proportional to storage, } \\
\text { drainage driven by gravity, } \\
\text { Penman-Monteith equation for ET } \\
\text { estimation, }\end{array}$ & $\begin{array}{l}\text { Four } \\
\text { layers }\end{array}$ & $\begin{array}{l}\text { Modified IGBP MODIS 20- } \\
\text { category vegetation, vegetation } \\
\text { fraction derived based on } \\
\text { Normalized Difference } \\
\text { Vegetation Index (NDVI) from } \\
\text { AVHRR, Soil parameters } \\
\text { derived from FAO. }\end{array}$ & $\begin{array}{l}\text { Precipitation, air temperature, } \\
\text { wind speed, surface pressure, } \\
\text { humidity, surface downward and } \\
\text { longwave radiations. }\end{array}$ \\
\hline CLM v3.0 & $\begin{array}{l}\text { TOPMODEL for surface runoff, } \\
\text { groundwater scheme, , Penman- } \\
\text { Monteith equation for ET }\end{array}$ & $\begin{array}{l}10 \\
\text { Layers }\end{array}$ & $\begin{array}{l}\text { Vegetation parameters } \\
\text { represented for } 17 \text { plant } \\
\text { functional types (Bonan et al. } \\
\text { 2002), soil parameters based on } \\
\text { IGBP. }\end{array}$ & $\begin{array}{l}\text { Precipitation, air temperature, } \\
\text { specific humidity, incident solar } \\
\text { radiation, and surface pressure. }\end{array}$ \\
\hline
\end{tabular}


Table S2: Performance of three LSMs (VIC, NOAH, and CLM) in terms of Nash Sutcliffe Efficiency (NS) and correlation coefficient (R) for monthly streamflow simulations compared against observations across 19 gauging stations across India (see Figure S1 for locations of these basins) for calibration and validation periods. Additionally the skill of the ensemble mean streamflow (ENS) from the three LSMs is also provided in this Table.

\begin{tabular}{|c|c|c|c|c|c|c|c|c|c|c|c|c|c|c|c|}
\hline \multirow{2}{*}{ No } & \multirow{2}{*}{ Basins } & \multirow{2}{*}{ Station } & \multirow{2}{*}{$\begin{array}{l}\text { Lon } \\
\left(^{\circ}\right)\end{array}$} & \multirow{2}{*}{$\begin{array}{l}\text { Lat } \\
\left({ }^{\circ}\right)\end{array}$} & \multirow{2}{*}{$\begin{array}{l}\text { Drainage } \\
\text { Area }\left(\mathbf{k m}^{2}\right)\end{array}$} & \multirow{2}{*}{\multicolumn{2}{|c|}{ Period }} & \multicolumn{2}{|l|}{ VIC } & \multicolumn{2}{|l|}{ Noah } & \multicolumn{2}{|c|}{ CLM } & \multicolumn{2}{|l|}{ ENS } \\
\hline & & & & & & & & $\mathbf{R}$ & NS & $\mathbf{R}$ & NS & $\mathbf{R}$ & NS & $\mathbf{R}$ & NS \\
\hline \multirow{2}{*}{$\mathrm{a}$} & \multirow{2}{*}{ Brahmani } & \multirow{2}{*}{ Anandpur } & \multirow{2}{*}{86.12} & \multirow{2}{*}{21.21} & \multirow{2}{*}{10,000} & Calibration & 1974-1982 & 0.93 & 0.86 & 0.89 & 0.63 & 0.92 & 0.59 & 0.95 & 0.78 \\
\hline & & & & & & Validation & 1985-1990 & 0.95 & 0.74 & 0.90 & 0.48 & 0.94 & 0.64 & 0.96 & 0.74 \\
\hline \multirow{2}{*}{$b$} & \multirow{2}{*}{ Brahmaputra } & \multirow{2}{*}{ Bahadpur } & \multirow{2}{*}{89.66} & \multirow{2}{*}{25.18} & \multirow{2}{*}{444,375} & Calibration & 1969-1971 & 0.88 & 0.66 & 0.96 & 0.90 & 0.90 & 0.76 & 0.94 & 0.85 \\
\hline & & & & & & Validation & 1973-1975 & 0.94 & 0.85 & 0.98 & 0.94 & 0.92 & 0.82 & 0.96 & 0.89 \\
\hline \multirow{2}{*}{ c } & \multirow{2}{*}{ Cauvery } & \multirow{2}{*}{ KMVadi } & \multirow{2}{*}{76.29} & \multirow{2}{*}{12.34} & \multirow{2}{*}{3125} & Calibration & 1991-1995 & 0.85 & 0.36 & 0.92 & -6.14 & 0.36 & $\begin{array}{l}- \\
0.46\end{array}$ & 0.85 & -4.34 \\
\hline & & & & & & Validation & 1996-2000 & 0.93 & -10.78 & 0.90 & -20.2 & 0.38 & $-\overline{1.40}$ & 0.89 & -7.92 \\
\hline \multirow{2}{*}{ d } & \multirow{2}{*}{ Ganga } & \multirow{2}{*}{ Farakka } & & & & Calibration & 1952-1960 & 0.98 & 0.89 & 0.98 & 0.93 & 0.88 & 0.73 & 0.98 & 0.92 \\
\hline & & & 87.92 & 24.83 & 865,000 & Validation & $1965-1973$ & 0.98 & 0.78 & 0.98 & 0.91 & 0.87 & 0.66 & 0.98 & 0.88 \\
\hline & & Polar & 8178 & & & Calibration & $1952-1960$ & 0.98 & 0.95 & 0.97 & 0.74 & 0.96 & 0.90 & 0.98 & 0.92 \\
\hline e & Godavarı & Polavaram & 81.78 & 16.92 & 260,000 & Validation & $1965-1973$ & 0.95 & 0.81 & 0.94 & 0.72 & 0.93 & 0.85 & 0.97 & 0.88 \\
\hline if & Fast coast & Tiruk & 7926 & 1298 & 10625 & Calibration & 1976-1979 & 0.79 & 0.30 & 0.85 & -2.20 & 0.69 & $\begin{array}{l}- \\
2.31 \\
\end{array}$ & 0.80 & -2.24 \\
\hline & Last cuast & 111 पूर & 17.20 & 12.00 & & Validation & 1978-1979 & 0.83 & -1.43 & 0.87 & 0.61 & 0.73 & $\begin{array}{l}- \\
0.72\end{array}$ & 0.85 & -0.13 \\
\hline $\mathrm{g}$ & North east & Purus & 84.87 & 19.51 & 6250 & Calibration & $1997-2000$ & 0.87 & 0.75 & 0.81 & 0.17 & 0.81 & $\begin{array}{l}- \\
0.09\end{array}$ & 0.86 & 0.36 \\
\hline & & & & & & Validation & $2001-2005$ & 0.94 & 0.78 & 0.93 & 0.70 & 0.87 & 0.45 & 0.95 & 0.78 \\
\hline h & & Thumn & 7670 & 923 & 1875 & Calibration & 1980-1990 & 0.79 & 0.58 & 0.83 & 0.62 & 0.88 & 0.52 & 0.91 & 0.82 \\
\hline $\mathrm{h}$ & South coast & I hump & 10.10 & 9.23 & $18 / 5$ & Validation & 1991-1995 & 0.89 & 0.53 & 0.94 & 0.01 & 0.65 & 0.39 & 0.91 & 0.67 \\
\hline $\mathrm{i}$ & West coast & Sante & 74.59 & 14.43 & 2500 & Calibration & 1989-1999 & 0.98 & 0.90 & 0.98 & 0.90 & 0.97 & 0.93 & 0.98 & 0.94 \\
\hline
\end{tabular}




\begin{tabular}{|c|c|c|c|c|c|c|c|c|c|c|c|c|c|c|c|}
\hline & & & & & & Validation & $2000-2005$ & 0.87 & 0.55 & 0.89 & 0.50 & 0.88 & 0.59 & 0.88 & 0.57 \\
\hline \multirow{2}{*}{ j } & \multirow{2}{*}{ Indus } & \multirow{2}{*}{ Baram } & \multirow{2}{*}{74.33} & \multirow{2}{*}{34.22} & \multirow{2}{*}{13,125} & Calibration & $1968-1974$ & 0.9 & 0.68 & 0.87 & 0.73 & 0.79 & 0.53 & 0.86 & 0.62 \\
\hline & & & & & & Validation & 1976-1979 & 0.90 & 0.80 & 0.84 & 0.68 & 0.88 & 0.71 & 0.84 & 0.69 \\
\hline \multirow{2}{*}{$\mathrm{k}$} & \multirow{2}{*}{ Krishna } & \multirow{2}{*}{ Takli } & \multirow{2}{*}{75.85} & \multirow{2}{*}{17.41} & \multirow{2}{*}{37,500} & Calibration & 1969-1974 & 0.88 & 0.76 & 0.90 & 0.77 & 0.82 & 0.67 & 0.91 & 0.81 \\
\hline & & & & & & Validation & 1976-1979 & 0.82 & 0.64 & 0.90 & 0.74 & 0.74 & 0.53 & 0.88 & 0.74 \\
\hline \multirow{2}{*}{1} & \multirow{2}{*}{ Mahanadi } & \multirow{2}{*}{ Basantpur } & \multirow{2}{*}{82.79} & \multirow{2}{*}{21.72} & \multirow{2}{*}{48,750} & Calibration & $1972-1980$ & 0.92 & 0.80 & 0.94 & 0.75 & 0.95 & 0.90 & 0.96 & 088 \\
\hline & & & & & & Validation & 1976-1980 & 0.95 & 0.88 & 0.94 & 0.84 & 0.96 & 0.88 & 0.97 & 0.93 \\
\hline \multirow{2}{*}{$\mathrm{m}$} & \multirow{2}{*}{ Mahi } & \multirow{2}{*}{ Khanpur } & \multirow{2}{*}{73.14} & \multirow{2}{*}{22.53} & \multirow{2}{*}{30,625} & Calibration & 1980-1995 & 0.96 & 0.93 & 0.97 & 0.93 & 0.85 & 0.68 & 0.94 & 0.84 \\
\hline & & & & & & Validation & 1996-2005 & 0.92 & 0.71 & 0.96 & 0.93 & 0.84 & 0.58 & 0.96 & 0.77 \\
\hline \multirow{2}{*}{ n } & \multirow{2}{*}{ Narmada } & \multirow{2}{*}{$\begin{array}{l}\text { Garudeshw } \\
\text { ar }\end{array}$} & \multirow{2}{*}{73.65} & \multirow{2}{*}{21.89} & \multirow{2}{*}{76,250} & Calibration & 1973-1987 & 0.97 & 0.93 & 0.97 & 0.93 & 0.97 & 0.93 & 0.98 & 0.97 \\
\hline & & & & & & Validation & 1996-2005 & 0.95 & 0.66 & 0.96 & 0.79 & 0.92 & 0.64 & 0.96 & 0.77 \\
\hline \multirow{2}{*}{ o } & \multirow{2}{*}{ Pennar } & & & & & Calibration & 1988-1989 & 0.93 & 0.85 & 0.85 & 0.72 & 0.92 & 0.84 & 0.93 & 0.87 \\
\hline & & Alladupall1 & 78.67 & 14.72 & $93 / 5$ & Validation & 1990-1991 & 0.89 & 0.70 & 0.83 & 0.65 & 0.84 & 0.62 & 0.91 & 0.83 \\
\hline & Saharmati & Ahmedaba & 7263 & 2308 & 10000 & Calibration & 1968-1969 & 0.90 & 0.69 & 0.96 & 0.93 & 0.95 & 0.43 & 0.95 & 0.87 \\
\hline $\mathrm{p}$ & sabarmat1 & & 12.63 & 23.08 & 10,000 & Validation & 1970-1972 & 0.89 & 0.22 & 0.89 & 0.75 & 0.95 & 0.57 & 0.94 & 0.69 \\
\hline & & & & & & Calibration & 1980-1986 & 0.97 & 0.93 & 0.97 & 0.92 & 0.92 & 0.84 & 0.97 & 0.93 \\
\hline $\mathrm{q}$ & Тар1 & Sarangpur & 74.53 & 21.43 & 46,250 & Validation & 1990-2005 & 0.93 & 0.81 & 0.91 & 0.83 & 0.88 & 0.76 & 0.93 & 0.85 \\
\hline r & Subarnarekh & Govindnur & 8607 & 2155 & 4375 & Calibration & 1994-2000 & 0.88 & 0.76 & 0.86 & 0.70 & 0.87 & 0.47 & 0.92 & 0.79 \\
\hline $\mathrm{r}$ & $\mathrm{a}$ & Govindpur & 86.92 & 21.55 & $43 / 5$ & Validation & $1990-2005$ & 0.94 & 0.83 & 0.92 & 0.84 & 0.81 & 0.35 & 0.92 & 0.81 \\
\hline
\end{tabular}


Table S3: Uncertainty in areal extent (\%) of area under severe to exceptional drought based on models simulated SSI during JJAS (shown in Figure 1a) and based on lagged 4-month SSI (shown in Figure 4) during major JJAS drought based on SPI

\begin{tabular}{|c|c|c|c|c|c|c|c|c|}
\hline Major JJAS drought (based on SPI) & \multicolumn{4}{|c|}{ JJAS SSI (Figure 1) } & \multicolumn{4}{|c|}{ Lagged 4-month SSI (Figure 4) } \\
\hline & VIC & NOAH & CLM & Ensemble & JASO VIC & JASO NOAH & ASON CLM & Ensemble \\
\hline 1987 & 37.74 & 39.16 & 33.05 & 36.73 & 35.70 & 38.93 & 32.45 & 35.92 \\
\hline 2002 & 34.11 & 38.73 & 37.65 & 35.95 & 38.98 & 48.54 & 37.97 & 41.42 \\
\hline 1979 & 26.61 & 26.25 & 13.19 & 19.87 & 30.31 & 27.28 & 21.15 & 25.49 \\
\hline 1972 & 32.54 & 47.44 & 19.21 & 29.81 & 32.71 & 39.03 & 24.43 & 30.27 \\
\hline 2009 & 29.05 & 34.20 & 33.56 & 31.46 & 22.45 & 27.70 & 29.03 & 26.13 \\
\hline 2015 & 14.01 & 21.21 & 18.13 & 16.51 & 24.33 & 32.82 & 29.07 & 27.51 \\
\hline
\end{tabular}

Table S4: Uncertainty in areal extent (\%) of area under severe to exceptional drought based on models simulated SSI during Rabi season (NDJF; shown in Figure 5) during major Rabi season drought based on Ensemble SSI.

\begin{tabular}{|c|c|c|c|c|}
\hline Major Rabi season drought (based on Ensemble SSI) & \multicolumn{4}{|c|}{ Rabi season SSI (Figure 5) } \\
\hline & VIC & NOAH & CLM Ensemble \\
\hline 1966 & 16.93 & 25.21 & 25.79 & 21.90 \\
\hline 1973 & 12.40 & 30.11 & 19.61 & 20.68 \\
\hline 2001 & 17.25 & 40.75 & 24.75 & 26.17 \\
\hline 2003 & 19.02 & 36.27 & 34.85 & 28.84 \\
\hline
\end{tabular}


Table S5: Shows JJAS drought intensity (SSI) for different areal-extent (\%, of India) and return period (in years) for different models. GEV distribution was fitted to JJAS SSI for different areal-extent for the period 1901-2015.

\begin{tabular}{|c|c|c|c|c|c|c|c|c|c|c|c|c|c|c|c|c|c|c|}
\hline \multirow{2}{*}{$\begin{array}{l}\text { Return } \\
\text { period } \\
\text { Areal } \\
\text { Extent } \\
\end{array}$} & \multicolumn{3}{|c|}{10} & \multicolumn{3}{|c|}{20} & \multicolumn{3}{|c|}{50} & \multicolumn{3}{|c|}{100} & \multicolumn{3}{|c|}{200} & \multicolumn{3}{|c|}{500} \\
\hline & VIC & NOAH & CLM & VIC & NOAH & CLM & VIC & NOAH & CLM & VIC & NOAH & CLM & VIC & NOAH & CLM & VIC & NOAH & CLM \\
\hline 2 & -2.89 & -3.52 & -3.00 & -3.16 & -3.98 & -3.36 & -3.48 & -4.57 & -3.83 & -3.72 & -5.02 & -4.19 & -3.94 & -5.46 & -4.55 & -4.22 & -6.05 & -5.04 \\
\hline 5 & -2.46 & -2.93 & -2.51 & -2.66 & -3.31 & -2.77 & -2.89 & -3.79 & -3.09 & -3.04 & -4.15 & -3.31 & -3.18 & -4.50 & -3.52 & -3.34 & -4.96 & -3.79 \\
\hline 10 & -2.15 & -2.47 & -2.14 & -2.32 & -2.79 & -2.32 & -2.53 & -3.20 & -2.54 & -2.66 & -3.50 & -2.67 & -2.78 & -3.78 & -2.80 & -2.91 & -4.15 & -2.94 \\
\hline 20 & -1.81 & -2.01 & -1.78 & -1.98 & -2.29 & -1.93 & -2.18 & -2.64 & -2.09 & -2.31 & -2.90 & -2.20 & -2.43 & -3.14 & -2.29 & -2.57 & -3.44 & -2.38 \\
\hline 30 & -1.58 & -1.73 & -1.55 & -1.76 & -2.00 & -1.70 & -1.97 & -2.33 & -1.85 & -2.10 & -2.56 & -1.95 & -2.22 & -2.78 & -2.03 & -2.37 & -3.06 & -2.12 \\
\hline 40 & -1.41 & -1.52 & -1.38 & -1.59 & -1.78 & -1.53 & -1.80 & -2.10 & -1.68 & -1.94 & -2.32 & -1.77 & -2.07 & -2.54 & -1.85 & -2.22 & -2.80 & -1.94 \\
\hline 50 & -1.25 & -1.35 & -1.23 & -1.44 & -1.60 & -1.38 & -1.66 & -1.91 & -1.53 & -1.80 & -2.13 & -1.62 & -1.94 & -2.34 & -1.70 & -2.10 & -2.60 & -1.78 \\
\hline 60 & -1.11 & -1.19 & -1.10 & -1.30 & -1.44 & -1.24 & -1.53 & -1.75 & -1.39 & -1.68 & -1.96 & -1.49 & -1.82 & -2.16 & -1.57 & -1.99 & -2.42 & -1.65 \\
\hline 70 & -0.98 & -1.05 & -0.97 & -1.17 & -1.30 & -1.11 & -1.40 & -1.59 & -1.27 & -1.56 & -1.80 & -1.36 & -1.72 & -2.00 & -1.44 & -1.90 & -2.25 & -1.53 \\
\hline 80 & -0.84 & -0.91 & -0.83 & -1.04 & -1.15 & -0.98 & -1.28 & -1.44 & -1.13 & -1.45 & -1.64 & -1.23 & -1.61 & -1.83 & -1.31 & -1.81 & -2.07 & -1.40 \\
\hline 90 & -0.70 & -0.77 & -0.69 & -0.90 & -1.00 & -0.84 & -1.15 & -1.28 & -0.99 & -1.33 & -1.47 & -1.08 & -1.50 & -1.65 & -1.16 & -1.71 & -1.88 & -1.25 \\
\hline 100 & -0.52 & -0.60 & -0.51 & -0.73 & -0.82 & -0.65 & -0.99 & -1.08 & -0.80 & -1.18 & -1.27 & -0.90 & -1.35 & -1.44 & -0.97 & -1.58 & -1.65 & -1.06 \\
\hline
\end{tabular}


Table S6: Parameters and confidence interval for GEV distribution fitted to JJAS 1951-2015 SSI estimated using different models for different areal extent (\%, of India).

\begin{tabular}{|c|c|c|c|c|c|c|c|c|c|c|}
\hline \multirow[b]{2}{*}{ areal extent } & \multirow[b]{2}{*}{ parameter } & \multicolumn{3}{|c|}{ VIC } & \multicolumn{3}{|c|}{ NOAH } & \multicolumn{3}{|c|}{ CLM } \\
\hline & & value & Lowe r CI & Higher CI & value & Lower CI & Higher CI & value & Lower CI & Higher CI \\
\hline \multirow[t]{3}{*}{ 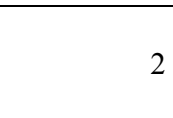 } & shape & -0.06 & -0.25 & 0.14 & 0.00 & -0.22 & 0.22 & 0.02 & -0.18 & 0.23 \\
\hline & scale & 0.42 & 0.35 & 0.52 & 0.63 & 0.51 & 0.78 & 0.47 & 0.38 & 0.58 \\
\hline & location & 2.00 & 1.88 & 2.12 & 2.10 & 1.92 & 2.28 & 1.92 & 1.79 & 2.05 \\
\hline \multirow{3}{*}{5} & shape & -0.14 & -0.35 & 0.06 & -0.02 & -0.25 & 0.21 & -0.06 & -0.26 & 0.13 \\
\hline & scale & 0.40 & 0.32 & 0.49 & 0.55 & 0.45 & 0.68 & 0.42 & 0.35 & 0.52 \\
\hline & location & 1.70 & 1.58 & 1.81 & 1.71 & 1.55 & 1.87 & 1.63 & 1.51 & 1.74 \\
\hline \multirow{3}{*}{10} & shape & -0.16 & -0.36 & 0.04 & -0.04 & -0.26 & 0.19 & -0.17 & -0.36 & 0.03 \\
\hline & scale & 0.38 & 0.31 & 0.46 & 0.50 & 0.40 & 0.62 & 0.40 & 0.33 & 0.49 \\
\hline & location & 1.43 & 1.33 & 1.54 & 1.39 & 1.25 & 1.53 & 1.39 & 1.28 & 1.50 \\
\hline \multirow{3}{*}{20} & shape & -0.15 & -0.34 & 0.03 & -0.06 & -0.27 & 0.15 & -0.22 & -0.38 & -0.07 \\
\hline & scale & 0.36 & 0.30 & 0.44 & 0.46 & 0.37 & 0.56 & 0.38 & 0.31 & 0.46 \\
\hline & location & 1.12 & 1.02 & 1.22 & 1.04 & 0.91 & 1.16 & 1.10 & 1.00 & 1.21 \\
\hline \multirow{3}{*}{30} & shape & -0.14 & -0.32 & 0.04 & -0.07 & -0.26 & 0.13 & -0.24 & -0.38 & -0.10 \\
\hline & scale & 0.36 & 0.29 & 0.43 & 0.44 & 0.36 & 0.54 & 0.38 & 0.31 & 0.45 \\
\hline & location & 0.90 & 0.80 & 0.99 & 0.80 & 0.68 & 0.93 & 0.90 & 0.80 & 1.00 \\
\hline \multirow{3}{*}{40} & shape & -0.13 & -0.31 & 0.04 & -0.07 & -0.26 & 0.12 & -0.24 & -0.38 & -0.11 \\
\hline & scale & 0.36 & 0.29 & 0.43 & 0.43 & 0.35 & 0.53 & 0.38 & 0.31 & 0.45 \\
\hline & location & 0.72 & 0.62 & 0.81 & 0.62 & 0.50 & 0.74 & 0.73 & 0.63 & 0.83 \\
\hline \multirow{3}{*}{50} & shape & -0.12 & -0.30 & 0.06 & -0.07 & -0.26 & 0.12 & -0.24 & -0.38 & -0.11 \\
\hline & scale & 0.35 & 0.29 & 0.43 & 0.42 & 0.35 & 0.52 & 0.38 & 0.31 & 0.45 \\
\hline & location & 0.56 & 0.46 & 0.65 & 0.47 & 0.35 & 0.58 & 0.58 & 0.48 & 0.68 \\
\hline \multirow{3}{*}{60} & shape & -0.11 & -0.30 & 0.08 & -0.07 & -0.26 & 0.11 & -0.24 & -0.37 & -0.11 \\
\hline & scale & 0.35 & 0.29 & 0.43 & 0.42 & 0.34 & 0.51 & 0.38 & 0.31 & 0.45 \\
\hline & location & 0.41 & 0.32 & 0.51 & 0.33 & 0.21 & 0.44 & 0.44 & 0.34 & 0.54 \\
\hline \multirow{3}{*}{70} & shape & -0.09 & -0.29 & 0.10 & -0.07 & -0.26 & 0.11 & -0.24 & -0.37 & -0.11 \\
\hline & scale & 0.35 & 0.28 & 0.42 & 0.41 & 0.34 & 0.50 & 0.38 & 0.31 & 0.45 \\
\hline & location & 0.27 & 0.18 & 0.37 & 0.20 & 0.09 & 0.32 & 0.31 & 0.21 & 0.41 \\
\hline \multirow{3}{*}{80} & shape & -0.08 & -0.28 & 0.12 & -0.08 & -0.26 & 0.11 & -0.24 & -0.37 & -0.10 \\
\hline & scale & 0.34 & 0.28 & 0.42 & 0.40 & 0.33 & 0.49 & 0.38 & 0.31 & 0.45 \\
\hline & location & 0.14 & 0.04 & 0.23 & 0.08 & -0.03 & 0.19 & 0.18 & 0.08 & 0.28 \\
\hline \multirow{3}{*}{90} & shape & -0.06 & -0.27 & 0.14 & -0.08 & -0.27 & 0.10 & -0.24 & -0.38 & -0.11 \\
\hline & scale & 0.33 & 0.27 & 0.41 & 0.40 & 0.33 & 0.48 & 0.38 & 0.31 & 0.45 \\
\hline & location & 0.00 & -0.10 & 0.09 & -0.04 & -0.15 & 0.07 & 0.04 & -0.06 & 0.14 \\
\hline \multirow{3}{*}{100} & shape & -0.05 & -0.26 & 0.17 & -0.09 & -0.28 & 0.10 & -0.24 & -0.38 & -0.11 \\
\hline & scale & 0.33 & 0.27 & 0.40 & 0.39 & 0.32 & 0.48 & 0.37 & 0.31 & 0.45 \\
\hline & location & -0.18 & -0.27 & -0.08 & -0.19 & -0.30 & -0.09 & -0.14 & -0.24 & -0.04 \\
\hline
\end{tabular}


Table S7: Shows NDJF drought intensity (SSI) corresponding to different areal-extent (\%, of India) and return period (in years) for different models. GEV distribution was fitted to NDJF SSI for different areal extent and for the period 1901-2015.

\begin{tabular}{|c|c|c|c|c|c|c|c|c|c|c|c|c|c|c|c|c|c|c|}
\hline \multirow{2}{*}{$\begin{array}{l}\text { Return } \\
\text { period } \\
\text { Areal } \\
\text { extent } \\
\end{array}$} & \multicolumn{3}{|c|}{10} & \multicolumn{3}{|c|}{20} & \multicolumn{3}{|c|}{50} & \multicolumn{3}{|c|}{100} & \multicolumn{3}{|c|}{200} & \multicolumn{3}{|c|}{500} \\
\hline & VIC & NOAH & CLM & VIC & NOAH & CLM & VIC & NOAH & CLM & VIC & NOAH & CLM & VIC & NOAH & CLM & VIC & NOAH & CLM \\
\hline 2 & -2.31 & -3.42 & -3.21 & -2.42 & -3.87 & -3.53 & -2.52 & -4.43 & -3.92 & -2.58 & -4.85 & -4.18 & -2.63 & -5.25 & -4.42 & -2.68 & -5.78 & -4.71 \\
\hline 5 & -1.99 & -2.90 & -2.71 & -2.08 & -3.26 & -2.96 & -2.17 & -3.71 & -3.25 & -2.23 & -4.02 & -3.44 & -2.27 & -4.31 & -3.60 & -2.31 & -4.68 & -3.80 \\
\hline 10 & -1.74 & -2.42 & -2.28 & -1.82 & -2.66 & -2.45 & -1.90 & -2.94 & -2.63 & -1.94 & -3.11 & -2.73 & -1.97 & -3.26 & -2.81 & -2.00 & -3.43 & -2.90 \\
\hline 20 & -1.48 & -1.94 & -1.85 & -1.55 & -2.11 & -1.97 & -1.62 & -2.28 & -2.07 & -1.65 & -2.37 & -2.13 & -1.68 & -2.45 & -2.17 & -1.70 & -2.52 & -2.22 \\
\hline 30 & -1.33 & -1.68 & -1.61 & -1.40 & -1.84 & -1.72 & -1.45 & -1.99 & -1.83 & -1.48 & -2.07 & -1.88 & -1.51 & -2.14 & -1.93 & -1.53 & -2.20 & -1.97 \\
\hline 40 & -1.21 & -1.49 & -1.42 & -1.27 & -1.64 & -1.54 & -1.33 & -1.78 & -1.65 & -1.36 & -1.87 & -1.71 & -1.38 & -1.93 & -1.76 & -1.40 & -1.99 & -1.80 \\
\hline 50 & -1.10 & -1.33 & -1.27 & -1.17 & -1.48 & -1.39 & -1.23 & -1.62 & -1.50 & -1.26 & -1.70 & -1.57 & -1.28 & -1.76 & -1.61 & -1.29 & -1.83 & -1.66 \\
\hline 60 & -1.01 & -1.18 & -1.13 & -1.08 & -1.33 & -1.25 & -1.14 & -1.47 & -1.37 & -1.17 & -1.55 & -1.44 & -1.19 & -1.62 & -1.49 & -1.21 & -1.68 & -1.54 \\
\hline 70 & -0.90 & -1.04 & -0.99 & -0.98 & -1.18 & -1.12 & -1.05 & -1.33 & -1.25 & -1.09 & -1.41 & -1.32 & -1.12 & -1.47 & -1.37 & -1.14 & -1.54 & -1.42 \\
\hline 80 & -0.80 & -0.90 & -0.86 & -0.88 & -1.04 & -0.99 & -0.96 & -1.18 & -1.12 & -1.00 & -1.27 & -1.19 & -1.03 & -1.33 & -1.25 & -1.06 & -1.39 & -1.31 \\
\hline 90 & -0.67 & -0.75 & -0.72 & -0.77 & -0.90 & -0.85 & -0.86 & -1.04 & -0.98 & -0.90 & -1.12 & -1.06 & -0.93 & -1.18 & -1.12 & -0.97 & -1.24 & -1.18 \\
\hline 100 & -0.51 & -0.59 & -0.54 & -0.61 & -0.72 & -0.68 & -0.70 & -0.86 & -0.81 & -0.75 & -0.94 & -0.89 & -0.78 & -1.00 & -0.95 & -0.82 & -1.06 & -1.01 \\
\hline
\end{tabular}


Table S8: Parameters and confidence interval for GEV distribution fitted to NDJF 1951-2015 SSI estimated for different areal-extent (\%, of India) using different models.

\begin{tabular}{|c|c|c|c|c|c|c|c|c|c|c|}
\hline \multirow[b]{2}{*}{$\begin{array}{l}\text { Areal } \\
\text { extent }\end{array}$} & \multirow[b]{2}{*}{ Parameter } & \multicolumn{3}{|c|}{ VIC } & \multicolumn{3}{|c|}{ NOAH } & \multicolumn{3}{|c|}{ CLM } \\
\hline & & Value & $\begin{array}{l}\text { Lower } \\
\text { CI }\end{array}$ & $\begin{array}{l}\text { Higher } \\
\text { CI }\end{array}$ & Value & $\begin{array}{l}\text { Lower } \\
\text { CI }\end{array}$ & $\begin{array}{l}\text { Higher } \\
\text { CI }\end{array}$ & Value & $\begin{array}{l}\text { Lower } \\
\text { CI }\end{array}$ & $\begin{array}{l}\text { Higher } \\
\text { CI }\end{array}$ \\
\hline \multirow{3}{*}{2} & Shape & -0.35 & -0.55 & -0.15 & -0.03 & -0.20 & 0.15 & -0.11 & -0.27 & 0.06 \\
\hline & Scale & 0.38 & 0.31 & 0.47 & 0.67 & 0.55 & 0.81 & 0.59 & 0.49 & 0.72 \\
\hline & Location & 1.72 & 1.62 & 1.82 & 1.96 & 1.78 & 2.15 & 2.03 & 1.86 & 2.19 \\
\hline \multirow{3}{*}{5} & Shape & -0.36 & -0.51 & -0.21 & -0.08 & -0.22 & 0.07 & -0.17 & -0.32 & -0.02 \\
\hline & Scale & 0.34 & 0.28 & 0.41 & 0.62 & 0.51 & 0.74 & 0.54 & 0.45 & 0.66 \\
\hline & Location & 1.47 & 1.38 & 1.56 & 1.63 & 1.46 & 1.79 & 1.69 & 1.54 & 1.84 \\
\hline \multirow{3}{*}{10} & Shape & -0.39 & -0.52 & -0.26 & -0.20 & -0.34 & -0.06 & -0.29 & -0.45 & -0.14 \\
\hline & Scale & 0.31 & 0.26 & 0.38 & 0.58 & 0.48 & 0.69 & 0.51 & 0.43 & 0.62 \\
\hline & Location & 1.27 & 1.19 & 1.35 & 1.37 & 1.21 & 1.53 & 1.43 & 1.29 & 1.57 \\
\hline \multirow{3}{*}{20} & Shape & -0.43 & -0.55 & -0.31 & -0.33 & -0.47 & -0.18 & -0.42 & -0.61 & -0.23 \\
\hline & Scale & 0.30 & 0.25 & 0.37 & 0.54 & 0.45 & 0.66 & 0.49 & 0.40 & 0.60 \\
\hline & Location & 1.05 & 0.97 & 1.13 & 1.08 & 0.93 & 1.22 & 1.13 & 1.00 & 1.27 \\
\hline \multirow{3}{*}{30} & Shape & -0.47 & -0.59 & -0.35 & -0.35 & -0.48 & -0.21 & -0.42 & -0.57 & -0.26 \\
\hline & Scale & 0.31 & 0.26 & 0.38 & 0.53 & 0.44 & 0.63 & 0.47 & 0.39 & 0.58 \\
\hline & Location & 0.89 & 0.81 & 0.98 & 0.86 & 0.72 & 1.00 & 0.91 & 0.79 & 1.04 \\
\hline \multirow{3}{*}{40} & Shape & -0.50 & -0.63 & -0.37 & -0.35 & -0.48 & -0.21 & -0.40 & -0.55 & -0.26 \\
\hline & Scale & 0.33 & 0.27 & 0.40 & 0.51 & 0.43 & 0.62 & 0.47 & 0.39 & 0.57 \\
\hline & Location & 0.76 & 0.68 & 0.85 & 0.69 & 0.55 & 0.83 & 0.73 & 0.61 & 0.86 \\
\hline \multirow{3}{*}{50} & Shape & -0.51 & -0.65 & -0.37 & -0.35 & -0.48 & -0.21 & -0.39 & -0.52 & -0.25 \\
\hline & Scale & 0.35 & 0.29 & 0.42 & 0.50 & 0.42 & 0.61 & 0.46 & 0.38 & 0.56 \\
\hline & Location & 0.64 & 0.54 & 0.73 & 0.54 & 0.41 & 0.67 & 0.58 & 0.46 & 0.70 \\
\hline \multirow{3}{*}{60} & Shape & -0.49 & -0.63 & -0.36 & -0.34 & -0.47 & -0.21 & -0.37 & -0.50 & -0.24 \\
\hline & Scale & 0.37 & 0.30 & 0.44 & 0.49 & 0.41 & 0.59 & 0.45 & 0.38 & 0.55 \\
\hline & Location & 0.51 & 0.41 & 0.61 & 0.40 & 0.27 & 0.53 & 0.44 & 0.32 & 0.56 \\
\hline \multirow{3}{*}{70} & Shape & -0.47 & -0.61 & -0.34 & -0.33 & -0.46 & -0.21 & -0.35 & -0.48 & -0.23 \\
\hline & Scale & 0.38 & 0.31 & 0.46 & 0.48 & 0.40 & 0.58 & 0.45 & 0.37 & 0.54 \\
\hline & Location & 0.38 & 0.28 & 0.48 & 0.27 & 0.14 & 0.40 & 0.30 & 0.18 & 0.42 \\
\hline \multirow{3}{*}{80} & Shape & -0.45 & -0.58 & -0.32 & -0.33 & -0.46 & -0.21 & -0.34 & -0.46 & -0.22 \\
\hline & Scale & 0.40 & 0.33 & 0.48 & 0.48 & 0.40 & 0.57 & 0.44 & 0.37 & 0.53 \\
\hline & Location & 0.24 & 0.13 & 0.34 & 0.14 & 0.02 & 0.27 & 0.17 & 0.05 & 0.28 \\
\hline \multirow{3}{*}{90} & Shape & -0.44 & -0.56 & -0.31 & -0.33 & -0.46 & -0.21 & -0.33 & -0.44 & -0.21 \\
\hline & Scale & 0.41 & 0.34 & 0.50 & 0.47 & 0.39 & 0.56 & 0.43 & 0.36 & 0.52 \\
\hline & Location & 0.09 & -0.02 & 0.19 & 0.01 & -0.11 & 0.14 & 0.03 & -0.08 & 0.15 \\
\hline \multirow{3}{*}{100} & Shape & -0.43 & -0.55 & -0.30 & -0.34 & -0.46 & -0.22 & -0.31 & -0.43 & -0.20 \\
\hline & Scale & 0.43 & 0.35 & 0.51 & 0.46 & 0.39 & 0.56 & 0.42 & 0.35 & 0.50 \\
\hline & Location & -0.11 & -0.22 & 0.00 & -0.14 & -0.27 & -0.02 & -0.14 & -0.25 & -0.03 \\
\hline
\end{tabular}


Table S9: Shows 12-month drought intensity (SSI) at the end of December corresponding to different areal-extent (\%, of Ganga region) and return period (in years) for different models. GEV distribution was fitted to 12-month SSI at the end of December for different areal extent and for the period 1901-2015.

\begin{tabular}{|c|c|c|c|c|c|c|c|c|c|c|c|c|c|c|c|c|c|c|}
\hline \multirow{2}{*}{$\begin{array}{l}\text { Return } \\
\text { period }\end{array}$} & \multicolumn{3}{|c|}{10} & \multicolumn{3}{|c|}{20} & \multicolumn{3}{|c|}{50} & \multicolumn{3}{|c|}{100} & \multicolumn{3}{|c|}{200} & \multicolumn{3}{|c|}{500} \\
\hline & VIC & NOAH & CLM & VIC & NOAH & CLM & VIC & NOAH & CLM & VIC & NOAH & CLM & VIC & NOAH & CLM & VIC & NOAH & CLM \\
\hline 2 & -2.96 & -3.60 & -2.79 & -3.20 & -4.07 & -3.04 & -3.45 & -4.64 & -3.30 & -3.61 & -5.02 & -3.46 & -3.74 & -5.37 & -3.60 & -3.88 & -5.80 & -3.75 \\
\hline 5 & -2.59 & -3.06 & -2.48 & -2.80 & -3.44 & -2.71 & -3.01 & -3.87 & -2.95 & -3.13 & -4.15 & -3.10 & -3.23 & -4.39 & -3.22 & -3.34 & -4.68 & -3.35 \\
\hline 10 & -2.28 & -2.63 & -2.24 & -2.46 & -2.95 & -2.44 & -2.65 & -3.31 & -2.65 & -2.75 & -3.53 & -2.77 & -2.83 & -3.71 & -2.87 & -2.92 & -3.92 & -2.97 \\
\hline 20 & -1.96 & -2.17 & -1.95 & -2.14 & -2.45 & -2.14 & -2.32 & -2.75 & -2.32 & -2.43 & -2.93 & -2.42 & -2.51 & -3.09 & -2.50 & -2.59 & -3.26 & -2.57 \\
\hline 30 & -1.75 & -1.88 & -1.75 & -1.94 & -2.15 & -1.93 & -2.13 & -2.42 & -2.10 & -2.24 & -2.59 & -2.20 & -2.32 & -2.74 & -2.27 & -2.42 & -2.89 & -2.34 \\
\hline 40 & -1.58 & -1.67 & -1.59 & -1.77 & -1.92 & -1.77 & -1.97 & -2.19 & -1.94 & -2.08 & -2.35 & -2.04 & -2.17 & -2.48 & -2.11 & -2.27 & -2.63 & -2.18 \\
\hline 50 & -1.42 & -1.49 & -1.45 & -1.62 & -1.74 & -1.63 & -1.82 & -1.99 & -1.80 & -1.94 & -2.15 & -1.90 & -2.03 & -2.28 & -1.97 & -2.13 & -2.42 & -2.04 \\
\hline 60 & -1.28 & -1.34 & -1.32 & -1.48 & -1.57 & -1.50 & -1.68 & -1.82 & -1.67 & -1.80 & -1.97 & -1.77 & -1.90 & -2.10 & -1.84 & -2.00 & -2.24 & -1.92 \\
\hline 70 & -1.15 & -1.19 & -1.19 & -1.34 & -1.42 & -1.37 & -1.55 & -1.67 & -1.54 & -1.67 & -1.82 & -1.64 & -1.77 & -1.94 & -1.72 & -1.88 & -2.08 & -1.80 \\
\hline 80 & -1.01 & -1.06 & -1.06 & -1.21 & -1.28 & -1.24 & -1.42 & -1.52 & -1.42 & -1.54 & -1.66 & -1.52 & -1.64 & -1.78 & -1.59 & -1.75 & -1.92 & -1.67 \\
\hline 90 & -0.88 & -0.92 & -0.92 & -1.07 & -1.14 & -1.10 & -1.28 & -1.37 & -1.28 & -1.40 & -1.51 & -1.38 & -1.51 & -1.63 & -1.45 & -1.62 & -1.75 & -1.53 \\
\hline 100 & -0.72 & -0.76 & -0.75 & -0.91 & -0.98 & -0.92 & -1.12 & -1.20 & -1.10 & -1.24 & -1.33 & -1.19 & -1.35 & -1.45 & -1.27 & -1.46 & -1.57 & -1.34 \\
\hline
\end{tabular}


Table S10: Parameters and confidence interval for GEV distribution fitted to 12-month SSI at the end of December for different areal-extent (\%, of Indo-Gangetic plain) for the period 1901-2015 simulated using different models.

\begin{tabular}{|c|c|c|c|c|c|c|c|c|c|c|}
\hline \multirow[b]{2}{*}{ Areal extent } & \multirow[b]{2}{*}{ Parameter } & \multicolumn{3}{|c|}{ VIC } & \multicolumn{3}{|c|}{ NOAH } & \multicolumn{3}{|c|}{ CLM } \\
\hline & & Value & $\begin{array}{l}\text { Lower } \\
\text { CI }\end{array}$ & $\begin{array}{l}\text { Higher } \\
\text { CI }\end{array}$ & Value & $\begin{array}{l}\text { Lower } \\
\text { CI }\end{array}$ & $\begin{array}{l}\text { Higher } \\
\text { CI }\end{array}$ & Value & $\begin{array}{l}\text { Lower } \\
\text { CI }\end{array}$ & $\begin{array}{l}\text { Higher } \\
\text { CI }\end{array}$ \\
\hline \multirow{3}{*}{2} & shape & -0.24 & -0.37 & -0.11 & -0.11 & -0.30 & 0.07 & -0.23 & -0.39 & -0.07 \\
\hline & scale & 0.63 & 0.53 & 0.76 & 0.89 & 0.73 & 1.09 & 0.63 & 0.52 & 0.76 \\
\hline & location & 1.86 & 1.69 & 2.03 & 1.83 & 1.58 & 2.07 & 1.69 & 1.52 & 1.86 \\
\hline \multirow{3}{*}{5} & shape & -0.29 & -0.42 & -0.16 & -0.17 & -0.35 & 0.00 & -0.26 & -0.42 & -0.10 \\
\hline & scale & 0.61 & 0.51 & 0.73 & 0.84 & 0.69 & 1.01 & 0.62 & 0.52 & 0.76 \\
\hline & location & 1.59 & 1.43 & 1.75 & 1.51 & 1.28 & 1.74 & 1.42 & 1.25 & 1.59 \\
\hline \multirow{3}{*}{10} & shape & -0.32 & -0.47 & -0.18 & -0.22 & -0.38 & -0.05 & -0.31 & -0.46 & -0.15 \\
\hline & scale & 0.59 & 0.49 & 0.71 & 0.79 & 0.65 & 0.95 & 0.64 & 0.53 & 0.78 \\
\hline & location & 1.33 & 1.17 & 1.49 & 1.23 & 1.01 & 1.44 & 1.19 & 1.02 & 1.37 \\
\hline \multirow{3}{*}{20} & shape & -0.32 & -0.48 & -0.17 & -0.24 & -0.41 & -0.08 & -0.36 & -0.52 & -0.20 \\
\hline & scale & 0.59 & 0.49 & 0.71 & 0.73 & 0.60 & 0.88 & 0.66 & 0.54 & 0.80 \\
\hline & location & 1.02 & 0.86 & 1.18 & 0.90 & 0.71 & 1.10 & 0.93 & 0.75 & 1.11 \\
\hline \multirow{3}{*}{30} & shape & -0.31 & -0.47 & -0.15 & -0.25 & -0.41 & -0.08 & -0.37 & -0.54 & -0.21 \\
\hline & scale & 0.59 & 0.49 & 0.71 & 0.69 & 0.57 & 0.84 & 0.66 & 0.55 & 0.81 \\
\hline & location & 0.79 & 0.64 & 0.95 & 0.69 & 0.50 & 0.88 & 0.74 & 0.56 & 0.92 \\
\hline \multirow{3}{*}{40} & shape & -0.30 & -0.46 & -0.14 & -0.25 & -0.41 & -0.08 & -0.37 & -0.54 & -0.21 \\
\hline & scale & 0.59 & 0.48 & 0.71 & 0.67 & 0.55 & 0.81 & 0.66 & 0.54 & 0.80 \\
\hline & location & 0.62 & 0.46 & 0.77 & 0.52 & 0.34 & 0.70 & 0.59 & 0.41 & 0.77 \\
\hline \multirow{3}{*}{50} & shape & -0.29 & -0.45 & -0.13 & -0.25 & -0.41 & -0.09 & -0.37 & -0.54 & -0.20 \\
\hline & scale & 0.58 & 0.48 & 0.70 & 0.65 & 0.54 & 0.78 & 0.65 & 0.54 & 0.79 \\
\hline & location & 0.46 & 0.31 & 0.62 & 0.38 & 0.20 & 0.55 & 0.45 & 0.27 & 0.63 \\
\hline \multirow{3}{*}{60} & shape & -0.28 & -0.44 & -0.12 & -0.25 & -0.41 & -0.09 & -0.36 & -0.53 & -0.19 \\
\hline & scale & 0.57 & 0.47 & 0.69 & 0.63 & 0.52 & 0.76 & 0.64 & 0.53 & 0.78 \\
\hline & location & 0.33 & 0.17 & 0.48 & 0.25 & 0.08 & 0.42 & 0.32 & 0.15 & 0.50 \\
\hline \multirow{3}{*}{70} & shape & -0.28 & -0.44 & -0.12 & -0.25 & -0.41 & -0.09 & -0.35 & -0.52 & -0.18 \\
\hline & scale & 0.57 & 0.47 & 0.68 & 0.61 & 0.51 & 0.74 & 0.63 & 0.52 & 0.77 \\
\hline & location & 0.20 & 0.04 & 0.35 & 0.14 & -0.03 & 0.30 & 0.20 & 0.03 & 0.37 \\
\hline \multirow{3}{*}{80} & shape & -0.27 & -0.43 & -0.11 & -0.25 & -0.40 & -0.09 & -0.35 & -0.52 & -0.18 \\
\hline & scale & 0.56 & 0.46 & 0.67 & 0.60 & 0.49 & 0.72 & 0.62 & 0.51 & 0.76 \\
\hline & location & 0.07 & -0.08 & 0.22 & 0.03 & -0.13 & 0.19 & 0.08 & -0.09 & 0.25 \\
\hline \multirow{3}{*}{90} & shape & -0.26 & -0.42 & -0.11 & -0.25 & -0.40 & -0.10 & -0.34 & -0.52 & -0.17 \\
\hline & scale & 0.55 & 0.45 & 0.66 & 0.58 & 0.48 & 0.70 & 0.61 & 0.50 & 0.74 \\
\hline & location & -0.05 & -0.20 & 0.10 & -0.08 & -0.24 & 0.08 & -0.04 & -0.21 & 0.13 \\
\hline \multirow{3}{*}{100} & shape & -0.26 & -0.42 & -0.10 & -0.25 & -0.41 & -0.10 & -0.34 & -0.52 & -0.17 \\
\hline & scale & 0.54 & 0.44 & 0.65 & 0.57 & 0.47 & 0.69 & 0.60 & 0.49 & 0.73 \\
\hline & location & -0.20 & -0.34 & -0.05 & -0.21 & -0.36 & -0.06 & -0.19 & -0.35 & -0.03 \\
\hline
\end{tabular}


Table S11: P (at 5\% significance level) and h value for goodness of fit-test (chi-square test) performed to test fit of GEV distribution fitted to monsoon season SSI for selected areal extents (\%) of drought in whole India. P $>0.05$ and $\mathrm{h}=0$ indicates that fit-test do not reject the null hypothesis that GEV distribution fits the monsoon season SSI for selected areal extent of drought in India.

\begin{tabular}{|r|r|r|r|r|r|r|}
\hline Areal Extents (\%) & \multicolumn{2}{|c|}{ VIC } & \multicolumn{2}{c|}{ NOAH } & \multicolumn{2}{c|}{ CLM } \\
\cline { 2 - 7 } & $\mathrm{p}$ & $\mathrm{h}$ & $\mathrm{p}$ & $\mathrm{h}$ & $\mathrm{p}$ & $\mathrm{h}$ \\
\hline 2 & 0.4561 & 0 & 0.1224 & 0 & 0.2017 & 0 \\
\hline 5 & 0.5614 & 0 & 0.0193 & 1 & 0.1253 & 0 \\
\hline 10 & 0.0039 & 1 & 0.1075 & 0 & 0.2826 & 0 \\
\hline 20 & 0.0895 & 0 & 0.4278 & 0 & 0.5560 & 0 \\
\hline 30 & 0.8090 & 0 & 0.4843 & 0 & 0.7565 & 0 \\
\hline 40 & 0.8653 & 0 & 0.6782 & 0 & 0.4341 & 0 \\
\hline 50 & 0.6169 & 0 & 0.9178 & 0 & 0.2901 & 0 \\
\hline 60 & 0.6535 & 0 & 0.8591 & 0 & 0.1416 & 0 \\
\hline 70 & 0.6797 & 0 & 0.9521 & 0 & 0.4647 & 0 \\
\hline 80 & 0.7765 & 0 & 0.6975 & 0 & 0.5668 & 0 \\
\hline 90 & 0.7715 & 0 & 0.8193 & 0 & 0.7120 & 0 \\
\hline 100 & 0.6236 & 0 & 0.5412 & 0 & 0.3994 & 0 \\
\hline
\end{tabular}


Table S12: P (at 5\% significance level) and h value for goodness of fit-test (chi-square test) performed to test fit of GEV distribution fitted to Rabi season SSI for selected areal extents (\%) of drought in whole India. P $>0.05$ and $\mathrm{h}=0$ indicates that fit-test do not reject the null hypothesis that GEV distribution fits the Rabi season SSI for selected areal extent of drought in India.

\begin{tabular}{|r|r|r|r|r|r|r|}
\hline Areal Extents (\%) & \multicolumn{2}{|c|}{ VIC } & \multicolumn{2}{c|}{ NOAH } & \multicolumn{2}{c|}{ CLM } \\
\cline { 2 - 7 } & $\mathrm{p}$ & $\mathrm{h}$ & $\mathrm{p}$ & $\mathrm{h}$ & $\mathrm{p}$ & $\mathrm{h}$ \\
\hline 2 & 0.0734 & 0 & 0.3150 & 0 & 0.5683 & 0 \\
\hline 5 & 0.5521 & 0 & 0.2178 & 0 & 0.2678 & 0 \\
\hline 10 & 0.1061 & 0 & 0.0437 & 1 & 0.8159 & 0 \\
\hline 20 & 0.5601 & 0 & 0.1862 & 0 & 0.0359 & 1 \\
\hline 30 & 0.0650 & 0 & 0.1159 & 0 & 0.4452 & 0 \\
\hline 40 & 0.3063 & 0 & 0.2194 & 0 & 0.2711 & 0 \\
\hline 50 & 0.4567 & 0 & 0.0423 & 1 & 0.2256 & 0 \\
\hline 60 & 0.7388 & 0 & 0.0592 & 0 & 0.2453 & 0 \\
\hline 70 & 0.8595 & 0 & 0.1084 & 0 & 0.7286 & 0 \\
\hline 80 & 0.6454 & 0 & 0.1711 & 0 & 0.6279 & 0 \\
\hline 90 & 0.1219 & 0 & 0.3509 & 0 & 0.5686 & 0 \\
\hline 100 & 0.5028 & 0 & 0.2988 & 0 & 0.4191 & 0 \\
\hline & & & \multicolumn{2}{c}{} &
\end{tabular}


Table S13: P (at 5\% significance level) and h value for goodness of fit-test (chi-square test) performed to test fit of GEV distribution fitted to annual SSI for selected areal extents (\%) of drought in Indo-Gangetic plain region. $\mathrm{P}>0.05$ and $\mathrm{h}=0$ indicates that fit-test do not reject the null hypothesis that GEV distribution fits the annual SSI for selected areal extent of drought in Indo-Gangetic plain region.

\begin{tabular}{|r|r|r|r|r|r|r|}
\hline Areal Extents (\%) & \multicolumn{2}{|c|}{ VIC } & \multicolumn{2}{c|}{ NOAH } & \multicolumn{2}{c|}{ CLM } \\
\cline { 2 - 7 } & $\mathrm{p}$ & $\mathrm{h}$ & $\mathrm{p}$ & $\mathrm{h}$ & $\mathrm{p}$ & $\mathrm{h}$ \\
\hline 2 & 0.4525 & 0 & 0.5415 & 0 & 0.3826 & 0 \\
\hline 5 & 0.7838 & 0 & 0.3348 & 0 & 0.7478 & 0 \\
\hline 10 & 0.0929 & 0 & 0.6391 & 0 & 0.9476 & 0 \\
\hline 20 & 0.1229 & 0 & 0.2932 & 0 & 0.8235 & 0 \\
\hline 30 & 0.1277 & 0 & 0.0367 & 1 & 0.6543 & 0 \\
\hline 40 & 0.0596 & 0 & 0.3943 & 0 & 0.6637 & 0 \\
\hline 50 & 0.0947 & 0 & 0.5460 & 0 & 0.7185 & 0 \\
\hline 60 & 0.0715 & 0 & 0.4994 & 0 & 0.6978 & 0 \\
\hline 70 & 0.2186 & 0 & 0.5070 & 0 & 0.5262 & 0 \\
\hline 80 & 0.1146 & 0 & 0.4389 & 0 & 0.6752 & 0 \\
\hline 90 & 0.0532 & 0 & 0.2795 & 0 & 0.6849 & 0 \\
\hline 100 & 0.1506 & 0 & 0.9097 & 0 & 0.8758 & 0 \\
\hline
\end{tabular}

